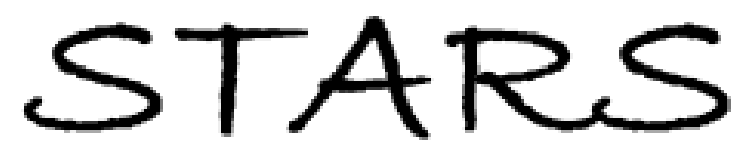

University of Central Florida

STARS

$1-1-2006$

\title{
Calculation of loosely bound levels for three-body quantum systems using hyperspherical coordinates with a mapping procedure
}

Viatcheslav Kokoouline

University of Central Florida

Françoise Masnou-Seeuws

Find similar works at: https://stars.library.ucf.edu/facultybib2000

University of Central Florida Libraries http://library.ucf.edu

This Article is brought to you for free and open access by the Faculty Bibliography at STARS. It has been accepted for inclusion in Faculty Bibliography 2000s by an authorized administrator of STARS. For more information, please contactSTARS@ucf.edu.

\section{Recommended Citation}

Kokoouline, Viatcheslav and Masnou-Seeuws, Françoise, "Calculation of loosely bound levels for threebody quantum systems using hyperspherical coordinates with a mapping procedure" (2006). Faculty Bibliography 2000s. 6309.

https://stars.library.ucf.edu/facultybib2000/6309

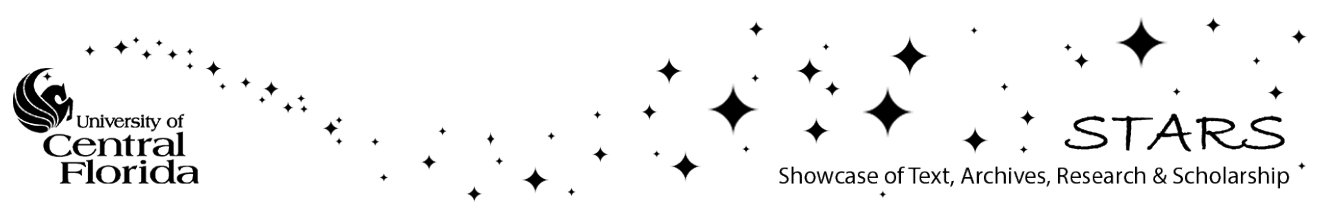




\title{
Calculation of loosely bound levels for three-body quantum systems using hyperspherical coordinates with a mapping procedure
}

\author{
Viatcheslav Kokoouline ${ }^{1,2}$ and Françoise Masnou-Seeuws ${ }^{2}$ \\ ${ }^{1}$ Department of Physics, University of Central Florida, Orlando, Florida 32816, USA \\ ${ }^{2}$ Laboratoire Aimé Cotton, CNRS, Bât. 505, Campus d'Orsay, 91405 Orsay Cedex, France
}

(Received 6 September 2005; published 10 January 2006)

\begin{abstract}
In view of modelization of experiments involving cold atoms and molecules, we develop a method that allows us to calculate weakly bound levels of triatomic molecules. The method combines (1) the hyperspherical coordinates to describe interparticle motion in the three-body system, (2) the solution of the Schrödinger equation in two steps: determination of adiabatic states for a fixed hyper-radius and then solution of a set of coupled hyper-radial equations using the slow variable representation of Tolstikhin et al. [J. Phys. B: At. Mol. Opt. Phys. 29, L389 (1996)], (3) and a mapping procedure that reduces considerably the number of basis functions needed to represent wave functions of weakly bound levels. We apply the method to the three different systems: the helium trimer ${ }^{4} \mathrm{He}_{3}$, isotopomers of the $\mathrm{H}_{3}{ }^{+}$ion, and finally a model three-body problem involving three nucleons. For all these systems, we show that the suggested method provides accurate results.
\end{abstract}

DOI: 10.1103/PhysRevA.73.012702

PACS number(s): 31.15.Ja, 02.70.Jn, 33.20.Tp

\section{INTRODUCTION}

The field of cold and ultracold molecules, which started less than ten years ago, is progressing rapidly [1]. Recent experiments on the formation of diatomic molecules in cold quantum gases [2-14], or in traps where a large atomic density is present [15-19], create physical situations where atom-dimer collisions play an important role. Accurate determination of atom-diatom collision data at ultracold temperatures is, therefore, a major problem and calculations are being developed by various groups in order to evaluate scattering lengths and vibrational quenching cross sections with sufficient accuracy $[1,20-26]$. Moreover, recent results [19] on Feshbach resonances for $\mathrm{Cs}_{2}$ molecules stored in a $\mathrm{CO}_{2}$ laser trap indicate the presence of bound $\mathrm{Cs}_{4}$ molecules, opening the way to the synthesis of complex molecules and the control of chemical reactions.

In particular, since both magnetic and optical Feshbach resonances have proved to be very efficient creating diatomic molecules out of two colliding ultracold atoms, it is expected that similar reactions could yield triatomic molecules starting from an atom-diatom colliding pair. Such molecules would be formed in loosely bound vibrational levels. Thus, there is a need for reliable methods to calculate the energies and wave functions of bound levels of triatomic systems, close to the dissociation limit. Like for the atom-diatom collision problem at ultralow energies, theoretical methods have to describe both an inner region where standard methods for triatomic molecules are applicable and an outer region extending up to hundreds of Bohr radii. In the asymptotic region of the potential surface, the vibrational motion is very slow, and hence the local de Broglie wavelength becomes very large, typically more than one order of magnitude larger than in the inner region. It is then convenient to perform numerical calculations using grid methods with a mapping procedure [27-33]. For loosely bound diatomic molecules, and for ultracold atom-atom collisions, the use of an adaptive coordinate following the variation of the local de Broglie wavelength has given very accurate results [28,29,33]. Therefore, the generalization to triatomic molecules and to atom-diatom collisions seems very timely.

Hyperspherical coordinates (HS), which were initially developed for nuclear reactions, are often used for the treatment of triatomic systems. They are well adapted to reactions where an insertion channel is present. For instance, the hyperspherical diabatic-by-sector method of Launay and coworkers [34] has been recently successful in predicting scattering lengths or vibrational quenching cross sections for ultracold $\mathrm{Na}+\mathrm{Na}_{2}[23]$ or $\mathrm{Li}+\mathrm{Li}_{2}[25,26]$ collisions, in situations where the spins of the three atoms are aligned. Another example is the determination of tightly bound vibrational levels of van der Waals molecules, such as $\mathrm{Ar}_{3}$ [35], where hyperspherical coordinates and hyperspherical adiabatic approximation were employed. The problem is then to generalize such methods to loosely bound triatomic molecules, where the vibrational motion extends to large distances. The aim of the present paper is to propose such a generalization, with a first application to the loosely bound level of ${ }^{4} \mathrm{He}_{3}$, and several vibrational levels of the four isopotomers of the $\mathrm{H}_{3}{ }^{+}$ion. Two aspects will therefore be considered.

(1) First, the implementation of a mapping procedure within the hyperspherical adiabatic approximation is described. The hyperspherical radius $\rho$ is changed to a new variable $x$ adapted to the local variation of the wave functions. This allows us to perform one-dimensional calculations up to large values of the hyper-radius.

(2) Second, in order to solve the system of onedimensional equations coupled by nonadiabatic couplings, we propose a method which is a modified version of the slow variable discretization introduced by Tolstikhin et al. [36].

The paper is organized as follows. In Sec. II we present the hyperspherical coordinates used in the present work. In Sec. III we describe the mapping procedure and the choice of the adaptive coordinate $x$. Section IV describes the slow variable discretization method. In Sec. V we report calculations 
for the ${ }^{4} \mathrm{He}_{3}$ trimer. In Sec. VI we report calculations for the four isotopomers of the $\mathrm{H}_{3}{ }^{+}$ion and discuss the accuracy of the adiabatic hyperspherical approach. Finally, in Sec. VII we discuss the similar problem of three nucleons. Section VIII is the Conclusion.

\section{HYPERSPHERICAL COORDINATES: ADIABATIC HYPERSPHERICAL APPROACH}

\section{A. Hyperspherical coordinates for three-body systems}

In the present treatment, we use the Smith-Whitten hyperspherical coordinates of Ref. [37]. Since several definitions exist in the literature (see, for example, Refs. [34,37-39]), we start by recalling the definition chosen here. Let $m_{i}$ with $i=1,2,3$ be masses of the three particles and $\vec{x}^{(i)}$ their radius vectors in the laboratory coordinate system. The following quantities are introduced first [37]:

$$
\begin{aligned}
& M=m_{1}+m_{2}+m_{3}, \\
& \mu=\sqrt{\frac{m_{1} m_{2} m_{3}}{M}}, \\
& d_{i}=\sqrt{\frac{m_{i}}{\mu}\left(1-\frac{m_{i}}{M}\right)}, \\
& \vec{r}^{(k)}=\frac{1}{d_{k}}\left(\vec{x}^{(j)}-\vec{x}^{(i)}\right) ; \quad i, j, k \text { are different, } \\
& \vec{R}^{(k)}=d_{k}\left[\vec{x}^{(k)}-\left(m_{j} \vec{x}^{(j)}+m_{i} \vec{x}^{(i)}\right) /\left(m_{j}+m_{i}\right)\right] ; \\
& i, j, k \text { are different, } \\
& \epsilon_{2}=2 \arctan \left(\frac{m_{3}}{\mu}\right), \\
& \epsilon_{3}=2 \arctan \left(\frac{m_{2}}{\mu}\right) .
\end{aligned}
$$

Then, using the above quantities, the three hyperspherical coordinates $\rho, \phi$, and $\theta$ are defined as

$$
\begin{gathered}
\rho^{2}=\left|\vec{r}^{(k)}\right|^{2}+\left|\vec{R}^{(k)}\right|^{2}, \\
\left|\vec{r}^{(1)}\right|=\frac{\rho d_{1}}{\sqrt{2}} \sqrt{1+\sin \theta \sin (\phi),} \\
\left|\vec{r}^{(2)}\right|=\frac{\rho d_{2}}{\sqrt{2}} \sqrt{1+\sin \theta \sin \left(\phi-\epsilon_{2}\right)}, \\
\left|\vec{r}^{(3)}\right|=\frac{\rho d_{3}}{\sqrt{2}} \sqrt{1+\sin \theta \sin \left(\phi+\epsilon_{3}\right)} .
\end{gathered}
$$

Although the hyper-radius $\rho$ is defined through $\vec{r}^{(k)}$ and $\vec{R}^{(k)}$, it does not depend on the choice of the index $k=1,2,3$. In the present treatment $\rho$ can vary in the interval $[0, \infty)$, the hyper-

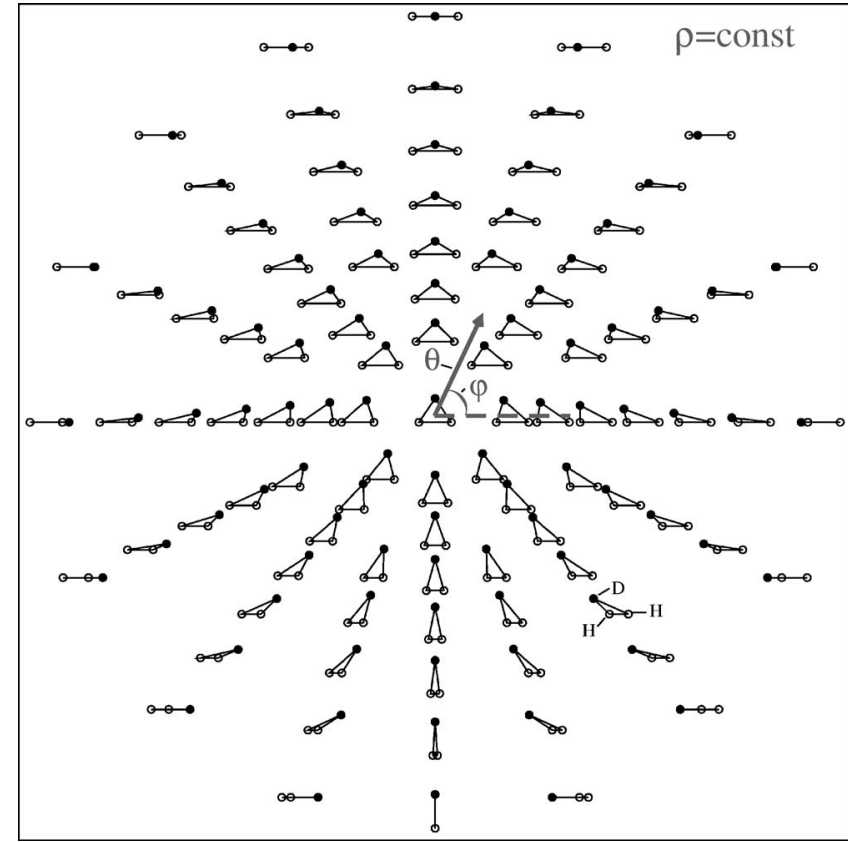

FIG. 1. The figure demonstrates how a two-dimensional space of the hyperangles $\theta$ and $\phi$ reproduces all possible shapes of a system like $\mathrm{H}_{2} \mathrm{D}^{+}$. Black circles symbolize $\mathrm{D}$, two white circles symbolize two hydrogen nuclei. This figure can be compared with Fig. (6) of Ref. [40], where all three nuclei are identical.

angle $\phi$ varies over $[0,2 \pi)$, and $\theta$ varies in the interval $[0, \pi / 2]$. The three coordinates can be used to describe the motion of three identical particles as well as nonidentical ones. Notice that the variation interval of $\phi$ is substantially different from a previous study, Ref. [39], where the helium trimer is also treated using the hyperspherical coordinates. Reference [39] uses $\varphi^{b}$, which is related to our $\phi$ as $\phi$ $=\pi / 2-2 \varphi^{b}$ and is adapted to a system of three identical particles, with a variation range $[-3 \pi / 4, \pi / 4]$. Moreover, in [39] the variation range of $\varphi^{b}$ is restricted to the interval $[0, \pi / 6](\varphi \in[\pi / 6, \pi / 2])$, which allows us to represent only a restricted set of possible eigenstates. Namely, with an appropriate choice of boundary conditions for $\varphi^{b}$ one can only represent vibrational eigenfunctions of the $A_{1}$ and $A_{2}$ irreducible representations of the $C_{3 v}$ vibrational group of three identical particles. Wave functions of the doubly degenerate irreducible $E$ representation cannot be obtained within the restricted interval for $\varphi^{b}$. Whereas this restriction has no consequence for the problem considered in Ref. [39], on the contrary, in the present work, we would like to calculate also the $E$ eigenstates. Moreover, in order to obtain vibrational eigenfunctions for three nonidentical particles, we have to use the whole range of the variation for $\phi$. As for the two other coordinates, $\rho$ is the same in Ref. [39], and the present study, while $\theta^{b}$ in Ref. [39] is related to our $\theta$ by $\theta=\pi /$ $-2 \theta^{b}$. In Refs. $[23,25,26]$ democratic hyperspherical adiabatic coordinates are used, which are slightly modified Smith-Whitten hyperspherical coordinates.

Figure 1 shows schematically all of the possible configurations of three particles as a function of the two hyperangles $\phi$ and $\theta$, for a fixed hyper-radius $\rho$. In the chosen example, 
masses are identical for two of the three particles, while the third mass is twice as large as the two others $\left(\mathrm{H}_{2} \mathrm{D}\right.$ molecule, for example).

\section{B. The adiabatic hyperspherical approximation}

In this work we consider only rotationless states of the three-body systems. Thus, the total angular momentum is zero.

The Schrödinger equation

$$
[T(\rho, \theta, \phi)+V(\rho, \theta, \phi)] \Phi_{n}(\rho, \theta, \phi)=E_{n}^{v i b} \Phi_{n}(\rho, \theta, \phi)
$$

for three particles interacting through the potential $V(\rho, \theta, \phi)$ in the hyperspherical coordinates is often solved in a twostep procedure. First, the adiabatic hyperspherical curves $U_{a}(\rho)$ and the corresponding hyperangular eigenstates $\varphi_{a}\left(\rho_{i} ; \theta, \phi\right)$ are obtained by a diagonalization in the twodimensional space of the hyperangles $\theta$ and $\phi, \rho$ being fixed at the value $\rho_{i}$ :

$$
H_{\rho_{i}}^{a d} \varphi_{a}\left(\rho_{i} ; \theta, \phi\right)=U_{a}\left(\rho_{i}\right) \varphi_{a}\left(\rho_{i} ; \theta, \phi\right) .
$$

In the above equation the adiabatic Hamiltonian $H_{\rho_{i}}^{\text {ad }}$ is the sum of the two terms: the grand angular momentum squared $\Lambda_{0}^{2}$ for zero angular momentum and the potential energy $V\left(\rho_{i} ; \theta, \phi\right)$,

$$
H_{\rho_{i}}^{a d}(\theta, \phi)=\hbar^{2} \frac{\Lambda_{0}^{2}+\frac{15}{4}}{2 \mu \rho_{i}^{2}}+V\left(\rho_{i} ; \theta, \phi\right) .
$$

The explicit form of the operator of the grand angular momentum squared is [41] (the total angular momentum is zero)

$$
\Lambda_{0}^{2}=-4\left[\frac{1}{\sin (2 \theta)} \frac{\partial}{\partial \theta} \sin (2 \theta) \frac{\partial}{\partial \theta}+\frac{1}{\sin ^{2} \theta} \frac{\partial^{2}}{\partial \phi^{2}}\right] .
$$

The solution of Eq. (4) yields adiabatic curves $U_{a}(\rho)$ and eigenfunctions $\varphi_{a}(\rho ; \theta, \phi)$, defining a set of adiabatic channels $a$.

The second step of the procedure is to solve a set of multichannel hyper-radial coupled Schrödinger equations. The three-dimensional eigenfunction $\Phi_{n}(\rho, \theta, \phi)$ is represented as a vector with components $\left(\psi_{1, n}, \psi_{2, n}, \ldots\right)$, corresponding to different adiabatic channels $a=1,2$; the equation reads

$$
\begin{gathered}
{\left[K(\rho)+U_{a}(\rho)\right] \psi_{a, n}(\rho)+\sum_{a^{\prime}}\left[W_{a, a^{\prime}} \psi_{a^{\prime}, n}(\rho)\right]} \\
=E_{n}^{v i b} \psi_{a, n}(\rho), \quad(n=1,2, \ldots),
\end{gathered}
$$

where $K(\rho)$ represents the kinetic energy term associated with hyper-radial motion

$$
K(\rho)=-\frac{\hbar^{2}}{2 \mu} \frac{d^{2}}{d \rho^{2}},
$$

and $W_{a, a^{\prime}}$ is the familiar nonadiabatic coupling element involving derivatives of $\varphi_{a^{\prime}}(\rho, \theta, \phi)$ with respect to $\rho$ (exact form can be found, for example, in Ref. [39]).

A numerical solution of the system of the coupled equations (7) provides exact solutions of the initial Schrödinger equation, Eq. (3). The grid method or the discrete variable representation (DVR) $[29,42-45]$, is an efficient tool in obtaining eigenenergies and the eigenfunctions of the Schrödinger equation. However, it is well known that Eq. (7) is difficult to solve due to the spiky dependence of the nonadiabatic couplings $W_{a, a^{\prime}}$ on the hyper-radius $\rho$. In order to obtain an accurate description of the spiky coupling terms, a very tight grid mesh along the $\rho$ axis has to be implemented. Because of this difficulty, one often uses the adiabatic approach, namely, one neglects the nonadiabatic couplings $W_{a, a^{\prime}}$.

The key idea of the adiabatic approach is to view the hyper-radial coordinate as a slowly varying coordinate in contrast to the rapidly changing hyperangular coordinates. This assumption is justified only if the couplings $W_{a, a^{\prime}}$ are small. Nevertheless, this assumption allows us to determine the adiabatic vibrational eigenenergies $E_{a, v}$ and the corresponding three-dimensional adiabatic vibrational wave functions $\Phi_{a, v}(\rho, \theta, \phi)$ by solving Eq. (7) for each potential $U_{a}(\rho)$ separately:

$$
\left[K(\rho)+U_{a}(\rho)-E_{a, v}\right] \psi_{a, v}(\rho)=0 .
$$

Correspondingly, assuming that all levels $n$ can be numbered by a pair of numbers $(a, v)$, where $a$ is the channel number and $v$ a vibrational number in this channel, the total threedimensional vibrational wave function and the eigenenergy in the adiabatic approximation are approximated as

$$
\begin{gathered}
\Phi_{n}(\rho, \theta, \phi) \approx \Phi_{a, v}(\rho, \theta, \phi)=\psi_{a, v}(\rho) \varphi_{a}(\rho ; \theta, \phi), \\
E_{n}^{v i b} \approx E_{a, v} .
\end{gathered}
$$

For numerical calculations, the hyper-radial wave functions $\psi_{a, v}(\rho)$ are represented as an expansion

$$
\psi_{a, v}(\rho)=\sum_{j=1}^{N} c_{j, a, v} \pi_{j}(\rho),
$$

on a set of $N$ basis functions $\pi_{j}(\rho)$. We have used two types of expansions, a DVR basis and a $B$-spline basis set. The solution of the Schrödinger equation Eq. (9) is then obtained by the diagonalization of a $N \times N$ matrix. In case of the DVR representation, $N$ is the number of grid points.

\section{MAPPING PROCEDURE}

\section{A. Change of the variable associated with the hyper-radius.}

When the energy of a bound level is close to the continuum (a weakly bound level), the corresponding wave function often extends to large interparticle distances. Weakly bound levels can be formed, for example, through Feshbach resonances in ultracold gases. Another example is the ground state of the $\mathrm{He}_{3}$ trimer, with a three-body bound level lying just $0.1 \mathrm{~K}$ below the continuum $[38,46]$, while the corresponding wave function becomes sufficiently small only at large interparticle distances.

In the numerical calculations, large distances between particles should be included into the treatment, leading to a large number $N$ of basis functions in the expansion of Eq. 
(11) and to diagonalization of a large $N \times N$ Hamiltonian matrix. In order to keep the size of the matrix reasonable, we use a mapping procedure to define a new set of basis functions that are better adapted to a given potential. We suggest to change not the basis functions $\pi_{j}(\rho)$ but the coordinate $\rho$ to a new one $x$, i.e., perform a mapping $\rho \rightarrow x(\rho)$. The mapping is made in such a way that in the new coordinate only a relatively small number of basis functions $\pi_{j}(x)$ is needed to represent the required eigenfunctions of the Hamiltonian.

One example where the mapping was particularly advantageous is the calculation of excited levels in diatomic systems $[29,33,47,48]$ using the DVR basis. For vibrational eigenfunctions $\psi(R)$ ( $R$ is the internuclear distance for the diatomic molecule) of weakly bound levels, the local de Broglie wavelength increases by more than one order of magnitude from short internuclear distances to the asymptotic region. This means that in the momentum representation eigenfunctions are not localized in a small region. In the coordinate representation, a very fine grid is required at short internuclear distances, but such a grid is superfluous at large distances. As a result, when a constant grid step is used, the DVR basis employed to represent accurately the eigenfunctions is unnecessarily large. Therefore a change of variable $R \rightarrow x$ is made in such a way that, in the new coordinate $x$, $\psi(x)$ has a local de Broglie wavelength that is approximately constant. The eigenfunction $\psi$ in the conjugated momentum $p_{x}$ representation is much better localized [29], leading to a smaller size of the basis of $\pi_{j}(x)$ functions that is needed to accurately represent the eigenfunctions. In the coordinate $R$ representation, this procedure produces a nonuniform DVR grid along $R$, which is more dense in the inner region where the weakly bound wave functions display many oscillations. In practice, the mapping $R(x)$ is estimated from the local de Broglie wavelength, which is obtained from the asymptotic kinetic energy and the potential $V(R)$ at given $R$. The mapping can be either analytical or numerical [29].

In the same way, the mapping can be performed, in the $B$-spline representation: the basis $B$-spline functions are not changed, but a new coordinate $x$ is introduced.

In this work we apply the mapping only along the hyperradial coordinate: we change the hyper-radius $\rho$ to the new coordinate $x: \rho=\rho(x)$. The grid steps $\Delta \rho$ and $\Delta x$ in $\rho$ and $x$ are linked by

$$
\Delta \rho=J(x) \Delta x, \quad \text { with } J(x)=\frac{d \rho}{d x} .
$$

In the coordinate $x$ the Schrödinger equation, Eq. (9), has the following form:

$$
\left[\tilde{K}(x)+U_{a}(\rho(x))\right] \phi_{a, v}(x)=E_{a, v} \phi_{a, v}(x),
$$

where we introduce a new wave function $\phi_{a, v}(x)$

$$
\phi_{a, v}(x)=\sqrt{J(x)} \psi_{a, v}(\rho(x)),
$$

which is normalized to 1 in the coordinate $x$

$$
\int\left|\phi_{a^{\prime}, v}(x)\right|^{2} d x=\int J(x)\left|\psi_{a^{\prime}, v}(\rho)\right|^{2} \frac{d \rho}{J(x)}=\int\left|\psi_{a^{\prime}, v}(\rho)\right|^{2} d \rho=1
$$

The kinetic energy term $\widetilde{K}(x)$ in the coordinate $x$ is

$$
\tilde{K}(x)=K(\rho(x))=\frac{\hbar^{2}}{4 \mu}\left[-\frac{1}{J^{2}} \frac{d^{2}}{d x^{2}}-\frac{d^{2}}{d x^{2}} \frac{1}{J^{2}}+\frac{7}{2} \frac{\left(J^{\prime}\right)^{2}}{J^{4}}-\frac{J^{\prime \prime}}{J^{3}}\right] .
$$

In the above equation, the derivatives $J^{\prime}$ and $J^{\prime \prime}$ are taken with respect to $x$.

\section{B. Numerical procedure}

The step $\Delta x$ is constant. For simplicity it can be chosen to be 1 . The step $\Delta \rho$ is variable and should be chosen according to the desirable grid density at a given region of $\rho$. Since for $\Delta x=1, J(x)=\Delta \rho(x)$, it is sufficient to determine the variable step $\Delta \rho$ for the whole interval of interest in order to obtain the functions $J(x)$ and $\rho(x)$. In practice, we perform the mapping procedure in the following way. We start with the smallest value $\rho_{1}$ of $\rho$ and determine the grid step $\Delta \rho_{1}$ according to a criterion that is appropriate to the required accuracy. The next point in the grid, $\rho_{2}$, is given by the sum $\rho_{1}+\Delta \rho_{1}$. Applying the accuracy criterion at $\rho_{2}$, we obtain $\Delta \rho_{2}$, then $\rho_{3}$ $=\rho_{2}+\Delta \rho_{2}$, and so on, until we reach the end of the interval of interest in $\rho$. The corresponding values of $x_{i}$ will be just $x_{i}$ $=i$.

The actual step $\Delta \rho$ at a given $\rho$ should be determined from the consideration of how dense the grid must be in that particular region. For example, if the wave function $\psi(\rho(x))$ in the vicinity of $\rho$ can be effectively represented by a few basis functions $\pi(x)$, the grid step $\Delta \rho=J(x)$ can be relatively large. As discussed above in Sec. III A, the wave functions for excited levels, which have many nodes, are well represented using the mapped DVR basis with the grid step linked to the local de Broglie wavelength

$$
\Delta \rho=\beta \frac{\pi}{\sqrt{2 \mu E_{\mathrm{kin}}(\rho)}} .
$$

$E_{\text {kin }}(\rho)$ in the above equation is the local kinetic energy calculated from the semiclassical consideration: at a given point $\rho, E_{\text {kin }}(\rho)=E_{\max }-U_{a}(\rho)$ is the difference between the maximum energy $E_{\max }$ considered in the calculation and the potential curve $U_{a}(\rho)$. The constant $\beta<1$ is introduced for a uniform control of the accuracy: the smaller the $\beta$, the more accurate the calculated eigenenergies and wave functions.

When several channels $a$ are involved (in the coupledchannel case), the same grid is used for all channels. The grid step can then be defined by considering the lowest potential curve at each $\rho$ value: $E_{\text {kin }}(\rho)=E_{\max }-\min \left(U_{a}(\rho)\right)$. A more convenient trick, especially when, in a diabatic representation, the potential curves $U_{a}(\rho)$ may cross, is to introduce an "enveloping potential": We define a smooth function $U_{\text {env }}(\rho)$ that "envelopes" all the potential curves $U_{a}(\rho)$ from below, lying at each $\rho$ below all of them. Then $E_{\text {kin }}(\rho)$ $=E_{\max }-U_{\text {env }}(\rho)$. 
By choosing the grid step in the way described above, we were able to calculate accurately hundreds of eigenstates of different diatomic molecules $[29,48]$ using a very modest DVR basis: the size of the basis was only a factor of 2 larger than the number of converged eigenenergies and wave functions $[29,48]$.

The procedure of determination of $\Delta \rho$ using $E_{\mathrm{kin}}(\rho)$ is efficient only for excited states having many nodes. If only the ground state or/and a few weakly excited states are needed, $\Delta \rho$ should be adapted to the local variation of the states.

In two recent studies, Refs. [49,50], there were attempts to optimize DVR bases for the three-body problem in order to reduce the number of grid points. In Ref. [49] the authors have used reduced one-dimensional classical Hamiltonians to construct an optimal grid. In Ref. [50] only boundaries of the grid in the three dimensions were optimized, not the grid sampling. In the present study, we optimize the grid in the $\rho$ dimension. The first step in the two-step diagonalization procedure can also be regarded as an optimization: from the complete basis of states $\varphi_{a}\left(\rho_{i} ; \theta, \phi\right)$ with $a=1, \ldots, \infty$, we chose only a few lowest states, which are then used to approximate the three-dimensional Hamiltonian.

\section{THE SLOW VARIABLE DISCRETIZATION METHOD: BEYOND THE ADIABATIC APPROXIMATION}

For many applications, the adiabatic hyperspherical approach is inaccurate, and one must include into consideration the nonadiabatic couplings $W_{a, a^{\prime}}$ between channels $a, a^{\prime}$. In the coordinate $x$ the Schrödinger equation, Eq. (7), has the following form:

$$
\left[\tilde{K}(x)+U_{a}(\rho(x))\right] \phi_{a, v}(x)+\sum_{a^{\prime}}\left[W_{a, a^{\prime}}(x) \phi_{a^{\prime}, v}(x)\right]=E_{n} \phi_{a, v}(x) .
$$

Because of the spiky character of the couplings $W_{a, a^{\prime}}(x)$, it is hard to represent them. At the same time, it is often desirable to keep the adiabatic coordinate $\rho$ and the two-step procedure for the solution of the Schrödinger equation described above. For example, in the theoretical study of the dissociative recombination of the $\mathrm{H}_{3}{ }^{+}$ion $[40,51,52]$ the hyper-radius naturally represents the dissociation coordinate. The slow variable discretization (SVD) method by Tolstikhin et al. [36] offers an opportunity to keep the hyper-radius as the dissociation coordinate and, at the same time, obtain essentially exact vibrational eigenfunctions.

The original detailed description of the SVD method is given in Ref. [36], where the method was applied to bound state calculations for a number of systems: $\operatorname{eep}\left(\mathrm{H}^{-}\right), \mathrm{dt} \mu$, and ppe $\left(\mathrm{H}_{2}^{+}\right)$. In our treatment, as described below, we have slightly modified the SVD method to be able to employ the basis $\left\{\pi_{j}(\rho)\right\}$ of $B$ splines.

First, the adiabatic eigenenergies $U_{a}\left(\rho_{i}\right)$ and eigenfunctions $\varphi_{a}\left(\rho_{i} ; \theta, \phi\right)$ at a fixed hyper-radius $\rho_{i}$ are obtained by solving the same eigenvalue equation, Eq. (4), as in the adiabatic approach. In a second step, the exact vibrational eigenstate $\Phi_{v i b}(\mathcal{Q})$ is represented as an expansion in the basis of the functions $\varphi_{a}\left(\rho_{i} ; \theta, \phi\right)$. The expansion coefficients $\psi_{a}\left(\rho_{i}\right)$ depend upon the hyper-radius $\rho_{i}$ :

$$
\Phi_{v i b}(\mathcal{Q})=\sum_{a} \psi_{a}\left(\rho_{i}\right) \varphi_{a}\left(\rho_{i} ; \theta, \phi\right)
$$

In Ref. [36] the hyper-radial wave functions $\psi_{a}\left(\rho_{i}\right)$ are then expanded in the DVR basis $\pi_{j}(\rho)$

$$
\psi_{a}(\rho)=\sum_{j} c_{j, a} \pi_{j}(\rho) .
$$

In the present treatment, for one application we use the original version of SVD with the DVR basis. However, for other applications discussed below, $B$-splines basis is more preferable. In fact, in the SVD method any space-localized basis can be used. Both, $B$-spline and DVR, basis functions are indeed localized in space. Inserting the two above expansions into the initial eigenvalue problem $H \Phi_{v i b}(\mathcal{Q})$ $=E \Phi_{v i b}(\mathcal{Q})$, we obtain

$$
\begin{aligned}
& \sum_{i^{\prime}, a^{\prime}}\left[\left\langle\pi_{i^{\prime}}|K(\rho)| \pi_{i}\right\rangle \mathcal{O}_{i^{\prime} a^{\prime}, i a}+\left\langle\pi_{i^{\prime}}\left|U_{a}(\rho)\right| \pi_{i}\right\rangle \delta_{a^{\prime} a}\right] c_{i^{\prime} a^{\prime}} \\
& \quad=E \sum_{i^{\prime}, a^{\prime}}\left\langle\pi_{i^{\prime}} \mid \pi_{i}\right\rangle \mathcal{O}_{i^{\prime} a^{\prime}, i a} c_{i^{\prime} a^{\prime}}
\end{aligned}
$$

with

$$
\mathcal{O}_{i^{\prime} a^{\prime}, i a}=\left\langle\varphi_{a^{\prime}}\left(\rho_{i^{\prime}} ; \theta, \phi\right) \mid \varphi_{a}\left(\rho_{i} ; \theta, \phi\right)\right\rangle .
$$

In Eq. (20), on the right-hand side the overlap matrix element $\left\langle\pi_{i^{\prime}} \mid \pi_{i}\right\rangle$ appears because the $B$-spline representation basis is not orthogonal. For the DVR basis, Eq. (20) is reduced to

$$
\begin{aligned}
& \sum_{i^{\prime}, a^{\prime}}\left[\left\langle\pi_{i^{\prime}}|K(\rho)| \pi_{i}\right\rangle \mathcal{O}_{i^{\prime} a^{\prime}, i a}+U_{a}\left(\rho_{i}\right) \delta_{i^{\prime}, i} \delta_{a^{\prime} a}\right] c_{i^{\prime} a^{\prime}} \\
& \quad=E \sum_{a^{\prime}} \mathcal{O}_{i a^{\prime}, i a} c_{i a^{\prime}} .
\end{aligned}
$$

The kinetic energy operator $K(\rho)$ contains second and, possibly, first derivatives with respect to $\rho$. In the above equation, the matrix elements $\left\langle\pi_{i^{\prime}}|K(\rho)| \pi_{i}\right\rangle$ can usually be calculated analytically (see, for example, [29,53], and references therein).

Equation (20) is written in the form of a generalized eigenvalue problem with the eigenvalue $E$ and the eigenvector $\vec{c}$. It can be solved using standard numerical procedures. The size of the matrices $H$ and $\mathcal{O}$ is $N \times M \times N \times M$, where $M$ is the number of adiabatic states $a$ in the expansion Eq. (18).

The advantage of the SVD method lies in a more efficient representation of the nonadiabatic coupling terms. The couplings $W_{a, a^{\prime}}(\rho)$ in the standard approach of Eq. (17) are replaced with the overlap matrix elements $\mathcal{O}_{i^{\prime} a^{\prime}, i a}$ between hyperangular adiabatic states $\varphi_{a}\left(\rho_{i} ; \theta, \phi\right)$ at different values of the hyper-radius. Using such representation of nonadiabatic couplings, the solution of the Schrödinger equation becomes easier to implement on the computer, since there is no need to calculate the first and second derivatives of the adiabatic 


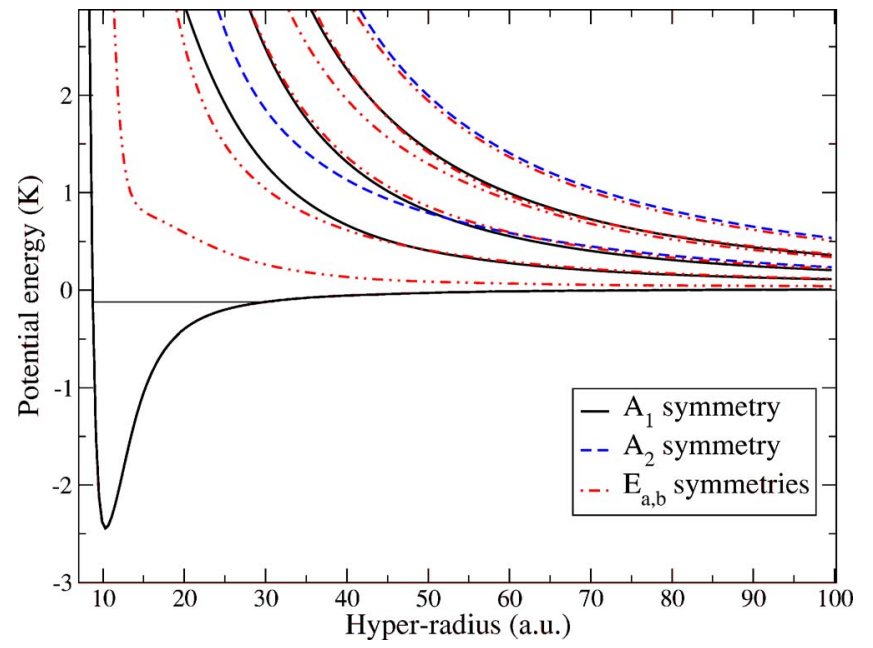

FIG. 2. (Color online) Several first adiabatic hyperspherical curves $U_{a}(\rho)$ of ${ }^{4} \mathrm{He}_{3}$. Every curve is classified according to one of the three irreducible representations of the $C_{3 v}$ vibrational group of $\mathrm{He}_{3}$. The $E$ representation is doubly degenerate; therefore, the curves for $E_{a}$ and $E_{b}$ coincide.

states $\varphi_{a}\left(\rho_{i} ; \theta, \phi\right)$. However, for an infinite number of grid points, the present method is equivalent to traditional methods using derivative nonadiabatic coupling.

The SVD method is similar in spirit to the diabatic-bysector method by Launay and co-workers $[23,25,26,34]$ : In both methods, for each hyper-radius, a separate $\rho$-adapted adiabatic $\varphi_{a}\left(\rho_{i} ; \theta, \phi\right)$ basis is calculated. However, in our opinion, such a $\rho$-adapted basis is utilized more efficiently in SVD. In the diabatic-by-sector method, a Numerov-type propagation procedure is implemented along each $\rho$ sector using the locally adapted adiabatic basis. In SVD, instead of the propagation, the locally adapted adiabatic bases are directly used to construct the global Hamiltonian matrix, which is then diagonalized. The result is that the locally adapted adiabatic hyperangular basis allows us to deal with a relatively small-sized Hamiltonian matrix.

\section{BOUND STATES CALCULATIONS FOR ${ }^{4} \mathrm{He}_{3}$}

A good system to test the method discussed above is the trimer ${ }^{4} \mathrm{He}_{3}$. The ground vibrational level is just about $0.1 \mathrm{~K}$ below the dissociation limit and, therefore, the corresponding vibrational wave function reaches out internuclear distances larger than 100 a.u. The energy of the level has been previously calculated $[38,39,46]$.

The previous calculations $[38,39,46]$ are based on the two potentials determined by Aziz and co-workers $[54,55]$. In the two references, the potentials, hereafter labeled as $V_{79}$ [54] and $V_{91}$ [55], are only slightly different. In the present work, we determine the bound vibrational states for both potentials and compare with the other calculations $[38,39,46]$.

We consider the ${ }^{4} \mathrm{He}$ isotope. The calculation is accomplished in two steps as discussed in the previous sections. In the first step, the adiabatic hyperspherical curves are calculated using Eq. (4). Several adiabatic curves are shown in Fig. 2. For the helium trimer involving identical isotopes, the

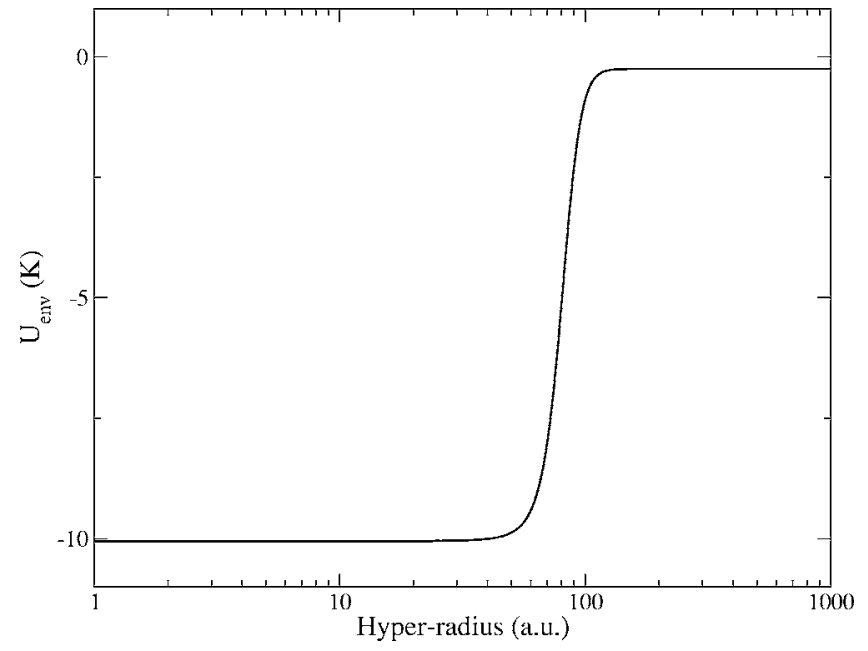

FIG. 3. Enveloping potential used for the mapping in the ${ }^{4} \mathrm{He}_{3}$ calculation.

vibrational levels are characterized according to one of the three irreducible representations, $A_{1}, A_{2}$, or $E$ of the $C_{3 v}$ symmetry group. Since the value of the hyper-radius does not influence the symmetry, the adiabatic hyperspherical curves can be characterized according to the same irreducible representations: the curves in Fig. 2 are classified accordingly.

In the second step, we obtain the actual energy of the vibrational levels by solving the Schrödinger equation in the one-dimensional hyper-radial space. We consider both, Eq. (7) that includes the nonadiabatic coupling terms $W_{a, a^{\prime}}$, and the adiabatic one, Eq. (9). For the numerical solution, we employ the DVR basis.

To test the mapping procedure, we use two types of grid along the $\rho$ coordinate: one with a constant grid step $\Delta \rho$ (no mapping), the second one with a variable step $\Delta \rho$ (with mapping). In the latter case, we use the enveloping potential shown in Fig. 3 to define the new coordinate $x$ and a grid step, which is relatively small for $\rho<60$ a.u. and larger for $\rho>60$ a.u.. We have to stress that in the calculation of the few lowest vibrational states, the optimal local grid step $\Delta \rho(\rho)$ cannot be easily determined from considerations of the local de Broglie wavelength. Looking at the adiabatic potential curves of Fig. 2 one could expect that the grid step should be increased starting from $\rho=30$ a.u., where no attractive potential is present any longer: however, our tests have shown that the dense grid must be kept until 60 a.u. This is explained by a strong nonadiabatic coupling between the lowest and the first excited adiabatic curves of the $A_{1}$ symmetry, displayed in Fig. 2 by the two lowest black solid curves. The lowest $A_{1}$ curve varies until 40 a.u., but the first excited $A_{1}$ curve varies significantly until 60 a.u.

Table I summarizes the results obtained for ${ }^{4} \mathrm{He}_{3}$ using the two available $\mathrm{He}$ dimer potentials $[54,55]$ and comparing with the previous calculations $[38,39,46]$. Cornelius and Glöckle [46] and Blume and Greene [39] calculated the energy only for one potential, while Esry et al. [38] computed the bound energies for both potentials.

In agreement with the work of Blume and Greene [39], we found that the nonadiabatic couplings play a considerable role: their inclusion increases the binding energy by $20 \%$. 
TABLE I. Vibrational binding energies of the ${ }^{4} \mathrm{He}_{3}$ trimer. Comparison between results from different calculations. Our calculation as well as the calculation of Ref. [38] is performed for the two available potentials [54,55]. Calculations of Refs. [46] and [39] were made using only one $\mathrm{He}_{2}$ potential.

\begin{tabular}{lllllll}
\hline \hline $\begin{array}{l}\mathrm{He}_{2} \\
\text { potential }\end{array}$ & Ref. [46] & Ref. [38] & Ref. [56] & Ref. [39] & $\begin{array}{l}\text { Present calc., } \\
\text { adiabatic approx. }\end{array}$ & $\begin{array}{l}\text { Present Calc., } \\
\text { SVD }\end{array}$ \\
\hline $\begin{array}{l}\text { Ref. [54] } \\
\text { Ref. [55] }\end{array}$ & $110 \mathrm{mK}$ & $98.11 \mathrm{mK}$ & & & $98 \mathrm{mK}$ & $115.5 \pm 0.5 \mathrm{mK}$ \\
\hline \hline
\end{tabular}

Table II illustrates in more detail the convergence of the eigenenergy with respect to the number of included $A_{1}$ states. Calculating the eigenenergy in the adiabatic representation, we have found the same result as Esry et al. [38] for the ground state. This was expected since Esry et al. [38] have used the same adiabatic approximation. The full calculation including the nonadiabatic couplings agrees better with the work by Cornelius and Glöckle [46] who used an alternative method. Blume and Greene [39] have included the nonadiabatic couplings using the familiar approach that involves derivatives $d / d \rho$ and $d^{2} / d \rho^{2}$ of the adiabatic states. Our result is in very good agreement with this calculation.

\section{VIBRATIONAL EIGENSTATES OF $\mathrm{H}_{3}{ }^{+}$AND ITS ISOTOPOMERS}

$\mathrm{H}_{3}{ }^{+}$is an important ion in interstellar clouds and atmospheres of large planets. It is also the simplest polyatomic molecule. A large number of theoretical articles are devoted to the ion. For our study it is important that vibrational energies of the ion are known with a great accuracy from experiments [57-59] and ab initio calculations [60-63]. The potential surface of the lowest molecular state $A_{1}^{\prime}$ of $\mathrm{H}_{3}{ }^{+}$was also determined with a great precision $[64,65]$ and can be used to calculate the vibrational energies of all four isotopomers of $\mathrm{H}_{3}{ }^{+}: \mathrm{H}_{3}{ }^{+}, \mathrm{D}_{3}{ }^{+}, \mathrm{H}_{2} \mathrm{D}^{+}$, and $\mathrm{D}_{2} \mathrm{H}^{+}$. In contrast to the two other three-body systems that we consider in this study, the $\mathrm{H}_{3}{ }^{+}$potential cannot be represented as a sum of three contributions from pair two-body interactions.

Similarly to $\mathrm{He}_{3}$, first, we have calculated the adiabatic hyperspherical curves. Figure 4 shows several such curves $U_{a}(\rho)$ for two isotopomers, $\mathrm{H}_{3}{ }^{+}$and $\mathrm{H}_{2} \mathrm{D}^{+}$. Curves for the other two isotopomers look similar. For comparison, we also give in Fig. 5 several $\mathrm{H}_{3}^{+}$and $\mathrm{H}_{2} \mathrm{D}^{+}$hyperangular wave functions $\varphi_{a}(\rho ; \theta, \phi)$ obtained solving the eigenvalues problem of Eq. (4). The vibrational wave functions of $\mathrm{H}_{3}{ }^{+}$belong to one of the three irreducible representations of the $C_{3 v}$ group: $A_{1}, A_{2}$, or $E$ ( $E$ is doubly degenerate). The wave functions of $\mathrm{H}_{2} \mathrm{D}^{+}$are classified according to $A$ or $B$ irreducible representations of the group $C_{2 v}$.

In the second step, we solve the hyper-radial Schrödinger equation using the SVD method. However, we did not use the DVR basis but the $B$-spline basis along $\rho$. Since we are interested only in the lowest vibrational states of the ions, we use a uniform grid: For the $\mathrm{H}_{3}{ }^{+}$isotopomers the mapping cannot be very effective because the wave functions are localized at small hyper-radii.

We calculated the vibrational energies for all four isotopomers. Table III compares vibrational energies obtained in the adiabatic hyperspherical approach with results obtained in the full three-dimensional calculation for four isotopomers of $\mathrm{H}_{3}{ }^{+}$. One can see that the adiabatic approach produces relatively accurate vibrational energies for $\mathrm{H}_{3}{ }^{+}$and $\mathrm{D}_{3}{ }^{+}$ions, but not for $\mathrm{H}_{2} \mathrm{D}^{+}$and $\mathrm{D}_{2} \mathrm{H}^{+}$. Adiabatic energies obtained for $\mathrm{H}_{3}{ }^{+}$are generally closer to the exact energies. The accuracy for $\mathrm{H}_{2} \mathrm{D}^{+}$and $\mathrm{D}_{2} \mathrm{H}^{+}$is worse because the symmetry of these ions is lower than in $\mathrm{H}_{3}{ }^{+}$and $\mathrm{D}_{3}{ }^{+}$. As a result, the adiabatic hyperspherical states interact stronger in $\mathrm{H}_{2} \mathrm{D}^{+}$and $\mathrm{D}_{2} \mathrm{H}^{+}$, and the adiabatic energies are less accurate.

Table IV compares the results of our SVD calculations (third column) for $\mathrm{H}_{2} \mathrm{D}^{+}$with a previous theoretical calculation [60] (fourth column). As one can see, agreement between the results is $0.2-0.7 \mathrm{~cm}^{-1}$. The second column in the table gives our result obtained using the adiabatic approximation and therefore neglecting the nonadiabatic coupling. The energies differ significantly from the results of the SVD calculation or previous study [60]. We attribute the poor accuracy of the adiabatic approximation for heteronuclear ions to the fact that the neglected nonadiabatic effects are stronger because the symmetry of the vibrational wave functions is lower. It means that more adiabatic states are mixed together and can contribute to a given vibrational level. This is illustrated in Fig. 6, which demonstrates the effect of the nonadiabatic couplings on a multichannel vibrational wave function of $\mathrm{H}_{2} \mathrm{D}^{+}$, corresponding to the first excited vibrational

TABLE II. Dependence of the binding energy (in $\mathrm{mK}$ ) of the ${ }^{4} \mathrm{He}_{3}$ ground vibrational level as a function of the number of adiabatic states $U_{a}$ included into the SVD calculation. For this calculation, the grid in the hyper-radius is equidistant and extended until 100 a.u. The number of hyper-radial grid points is 64 . For the comparison, we give also the results from Ref. [39], which also accounted for the nonadiabatic couplings.

\begin{tabular}{lllllll}
\hline \hline $\begin{array}{l}\text { Number of included adiabatic } \\
\text { states }\end{array}$ & 1 & 2 & 3 & 4 & 5 & 6 \\
\hline Results from Ref. [39] & 106 & 119 & 122 & 124 & 124 & 125 \\
Our results & 105 & 119 & 121.0 & 123.0 & 123.4 & 123.8 \\
\hline \hline
\end{tabular}



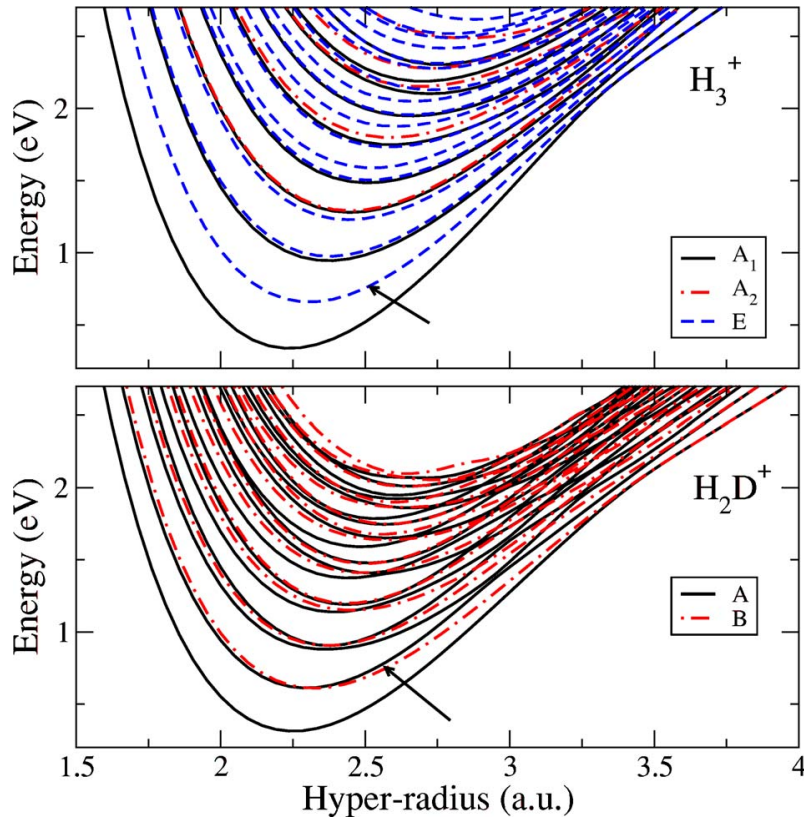

FIG. 4. (Color online) The figure shows several first adiabatic hyperspherical potential curves for the $\mathrm{H}_{3}{ }^{+}$and $\mathrm{H}_{2} \mathrm{D}^{+}$ions. The curves for $\mathrm{H}_{3}{ }^{+}$are classified according to one of the three, $A_{1}, E$, or $A_{2}$, irreducible representations of the vibrational symmetry group $C_{3 v}$ of $\mathrm{H}_{3}{ }^{+} ; \mathrm{H}_{2} \mathrm{D}^{+}$curves are classified according to one of the two, $A$ or $B$, representations of the $C_{2 v}$ group. Arrows in the two panels show how an $E$ doubly degenerate state in $\mathrm{H}_{3}{ }^{+}$splits into two different states in $\mathrm{H}_{2} \mathrm{D}^{+}$.

level $v_{A}=1,\left(v_{1} v_{2} v_{3}\right)=(010), E=2205.2 \mathrm{~cm}^{-1}$ of the $A$ symmetry. The figure shows components of the first three adiabatic channels of the $A$ irreducible representation. Clearly, the three components contribute significantly to the total wave function. Since in the adiabatic approximation the contribution from only one component is accounted for, it leads to a very poor accuracy of wave functions and eigenenergies.

\section{BENCHMARK THREE-BODY SYSTEM OF THREE NUCLEONS}

The third three-body problem that we consider in this study is the model benchmark system of three identical bosons (see Ref. [66] and references therein). The mass of the bosons is $m_{1}=m_{2}=m_{3}=1837.5773$ a.u. or equally 939 MeV. Similarly to $\mathrm{He}_{3}$, the three-body potential $V\left(r_{12}, r_{23}, r_{31}\right)$ is constructed as a sum of two-body pair potentials: $\quad V\left(r_{12}, r_{23}, r_{31}\right)=V_{2}\left(r_{12}\right)+V_{2}\left(r_{23}\right)+V_{2}\left(r_{31}\right)$, where $V_{2}(r)$ is in units of $\mathrm{MeV}$ has the following form:

$$
V_{2}(r)=-55 e^{-0.2 r^{2}}+1.5 e^{-0.01(r-5)^{2}},
$$

the distance $r$ is in fm. (in atomic units, 1 a.u. of length is $5.291772 \times 10^{-11} \mathrm{~m}, 1$ a.u. of energy is $27.721138 \mathrm{eV}$ ).

Since the two-body potential is constant at small distances, $<0.1 \mathrm{fm}$, the three-body potential for a fixed small hyper-radius, $\rho<0.1 \mathrm{fm}$, does not depend on the two hyperangles. The solutions of the hyperangular equation [Eq. (4)] for such small $\rho$ are just eigenstates of the grand angular momentum squared $\Lambda_{0}^{2}$, Eq. (6). These are hyperspherical harmonics with eigenvalues $\lambda(\lambda+4)$ [67]. To be able to compare with the analytic solution of Eq. (4), we demonstrate in Fig. 7 the adiabatic hyperspherical curves scaled in the way that gives eigenvalues of $\Lambda_{0}^{2}$ at small $\rho$, i.e., Fig 7 shows not the curves $U_{a}(\rho)$ themselves, but $u_{a}(\rho)=2 \mu \rho^{2} U_{a}(\rho)-\frac{15}{4}$. At small hyper-radii, each curve $U_{a}(\rho)$ correlates with one eigenvalue $\lambda(\lambda+4)$ of $\Lambda_{0}^{2}$. $\lambda$ can have only even values, $\lambda=0$, $2,4, \ldots$. In the present study we have only even $\lambda$ because we are considering a half of the hyperangular sphere, the second half can be represented by an extension of the hyperangle $\phi$ interval to $4 \pi$ [41]. The state with eigenvalue $\lambda(\lambda$ +4 ) has degeneracy $\lambda / 2+1$ at small $\rho$. At larger $\rho$ the symmetry is lower and each manifold with a given $\lambda$ splits into the states that are classified according to one of the three irreducible representations of the $C_{3 v}$ vibrational group: $A_{1}$, $A_{2}$, and $E$. The $E$ states are still doubly degenerate even at large $\rho$. The use of the $\phi$ interval from 0 to $2 \pi$ allows us to represent the three possible irreducible representations of the $C_{3 v}$ group. In contrast to the present study, in several previous studies $[38,39,66]$, the interval of $\phi$ is smaller and the boundary condition at the ends of the interval are chosen in such a way that only $A_{1}$ states are represented. As a result, in these studies the eigenvalue with $\lambda=2$ does not exist. Since the ground vibrational state, that we are interested in, is an $A_{1}$ state, such a reduced interval in $\phi$ in Refs. $[38,39,66]$ should give the same result as the present treatment. Vibrational states of other irreducible representations would be needed if some excited vibrational states have to be found.
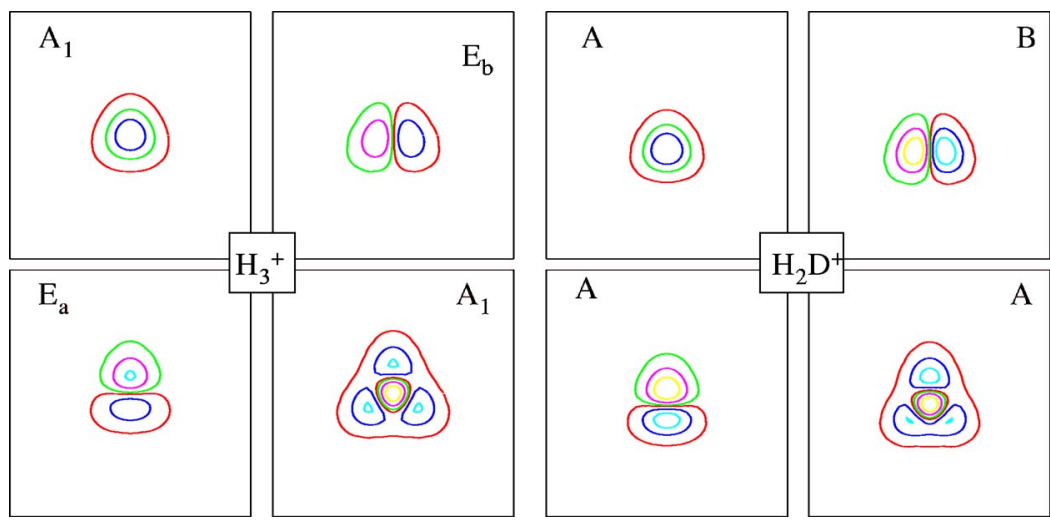

FIG. 5. (Color online) The figure shows wave functions of several hyperangular adiabatic states $\varphi_{a}(\rho ; \theta, \phi)$ for a fixed hyper-radius $\rho=2.2$ a.u. The four left panels give the wave functions obtained for the $\mathrm{H}_{3}{ }^{+}$ion; other panels represent the $\mathrm{H}_{2} \mathrm{D}^{+}$wave functions. All the wave functions are classified according to the irreducible representations of the corresponding vibrational symmetry group: For $\mathrm{H}_{3}{ }^{+}$the group is $C_{3 v}$, for $\mathrm{H}_{2} \mathrm{D}^{+}$it is $C_{2 v}$. The $E_{a}$ and $E_{b}$ irreducible representations of $\mathrm{H}_{3}{ }^{+}$are degenerate; any linear combination of them is also an eigenfunction of Eq. (4). 
TABLE III. Several vibrational energies of the four isotopomers of the $\mathrm{H}_{3}{ }^{+}$ion: $\mathrm{H}_{3}{ }^{+}, \mathrm{H}_{2} \mathrm{D}^{+}, \mathrm{D}_{2} \mathrm{H}^{+}$, and $\mathrm{D}_{3}{ }^{+}$. The first number in each cell of the table gives the energy obtained in the present study using the adiabatic approximation, the second number in each cell gives the energy of the state calculated in previous calculations. Accuracy of calculations in Refs. $[60,61,65]$ is better than $0.1 \mathrm{~cm}^{-1}$. All energies are given in $\mathrm{cm}^{-1}$ and are related to the ground vibrational level of the corresponding isotopomer.

\begin{tabular}{llllll}
\hline \hline$\left(v_{1} v_{2}^{l_{2}}\right), D_{3 h}$ & $\mathrm{H}_{3}^{+}$ & $\mathrm{D}_{3}^{+}$ & $\left(v_{1} v_{2} v_{3}\right), C_{2 v}$ & $\mathrm{H}_{2} \mathrm{D}^{+}$ & $\mathrm{D}_{2} \mathrm{H}^{+}$ \\
\hline$\left(10^{0}\right)$ & 3188 & 2306 & $(100)$ & 2781 & 2431 \\
& $3178.5^{\mathrm{a}}$ & $2301.36^{\mathrm{b}}$ & & $2992.51^{\mathrm{c}}$ & $2736.98^{\mathrm{c}}$ \\
$\left(01^{1}\right)$ & 2516 & 1833 & $(010)$ & 2382 & 2210 \\
& $2521.20^{\mathrm{a}}$ & $1834.67^{\mathrm{b}}$ & & $2205.87^{\mathrm{c}}$ & $1968.17^{\mathrm{c}}$ \\
$\left(02^{2}\right)$ & 5001 & 3650.8 & $(001)$ & 2328 & 2086 \\
& $4997.73^{\mathrm{a}}$ & $3650.55^{\mathrm{b}}$ & & $2335.45^{\mathrm{c}}$ & $2078.43^{\mathrm{c}}$ \\
\hline \hline
\end{tabular}

${ }^{\mathrm{a}}$ Reference [65].

${ }^{\mathrm{b}}$ Reference [61].

${ }^{\mathrm{c}}$ Reference [60].

We calculated the energy of the only bound $A_{1}$ state of the $3 n$ system using the $B$-spline basis along $\rho$. With the use of a uniform 256 point grid and the largest $\rho=160 \mathrm{fm}$, we obtained the energy $-37.216 \mathrm{MeV}$. This is in good agreement with the result of Ref. [66], -37.221 . We also tried to use the DVR basis along with the mapping. We employed a mapped grid with 64 points, while the largest $\rho$ was also $160 \mathrm{fm}$. The obtained bound energy is $-37.20 \mathrm{MeV}$. In these calculations, with and without the mapping, we used five adiabatic hyperspherical channels of the $A_{1}$ irreducible representation.

\section{SUMMARY AND CONCLUSIONS}

The main goal of the present study was to develop an accurate method that is general enough to be applied to a variety of three-body systems. In particular, the method can be used for loosely bound states of triatomic molecules, relevant to experiments with ultracold molecules.

We use the hyperspherical coordinates. The wave functions are expanded on a basis set, using either DVR or $B$-spline representation. Special attention in this study was paid to the problem of weakly bound states with wave functions reaching large interparticle separations. In order to keep the representation basis along the hyper-radius as small as possible, we suggested using the mapping procedure. The mapping procedure is very similar to the mapping for diatomic molecules $[29,47,48]$, where the variable change for the mapping was deduced from the local de Broglie wave- length. The actual solution of the Schrödinger equation for the three-body is made in two steps: (1) the adiabatic hyperspherical states and energies are calculated, (2) and a system of coupled one-dimensional hyper-radial equations is solved. To overcome the adiabatic approximation and avoid using derivatives of the adiabatic eigenstates with respect to the hyper-radius, we use the SVD method by Tolstikhin [36], using one of the two representation bases: DVR and $B$-splines. The calculation of derivatives is then replaced by overlap integrals, which is easier to implement numerically.

We consider two problems, where the use of the mapping was helpful: bound states of ${ }^{4} \mathrm{He}_{3}$ and a model problem of three nucleons. In both problems the ground bound state is just below the dissociation limit such that the corresponding wave functions are extended to large interparticle distances. We obtained the same result with and without the mapping for the two three-body systems. In the third application, isotopomers of $\mathrm{H}_{3}{ }^{+}$, the mapping was not employed because the wave functions of interest are localized at small hyper-radii.

For all the three three-body systems, we have found that the inclusion of the nonadiabatic hyperspherical couplings is important for the accuracy of the calculation. This is especially important where the three particles are not identical, because the symmetry of such a system is lower, the couplings are stronger, and the accuracy of the adiabatic approximation becomes worse. We have shown that such situation occurs for the isotopomers of $\mathrm{H}_{3}{ }^{+}$: the accuracy of the adiabatic approximation in $\mathrm{H}_{3}{ }^{+}$and $\mathrm{D}_{3}{ }^{+}$is much better than in $\mathrm{H}_{2} \mathrm{D}^{+}$and $\mathrm{D}_{2} \mathrm{H}^{+}$. For the helium trimer ${ }^{4} \mathrm{He}_{3}$, the inclusion

TABLE IV. Several vibrational energies of $\mathrm{H}_{2} \mathrm{D}^{+}$calculated using the adiabatic hyperspherical approximation and SVD method. For a comparison, the energies from the accurate calculation of Ref. [60] are also given.

\begin{tabular}{llll}
\hline \hline Symmetry, $\left(v_{1} v_{2} v_{3}\right)$ & Adiabatic energies & SVD energies & Previous calc. \\
\hline A, $(000)$ & 0 & 0 & 0 \\
A, $(100)$ & 2781 & 2993.8 & 2992.51 \\
A, $(010)$ & 2382 & 2205.2 & 2205.87 \\
B, $(001)$ & 2328 & 2335.2 & 2335.45 \\
\hline \hline
\end{tabular}




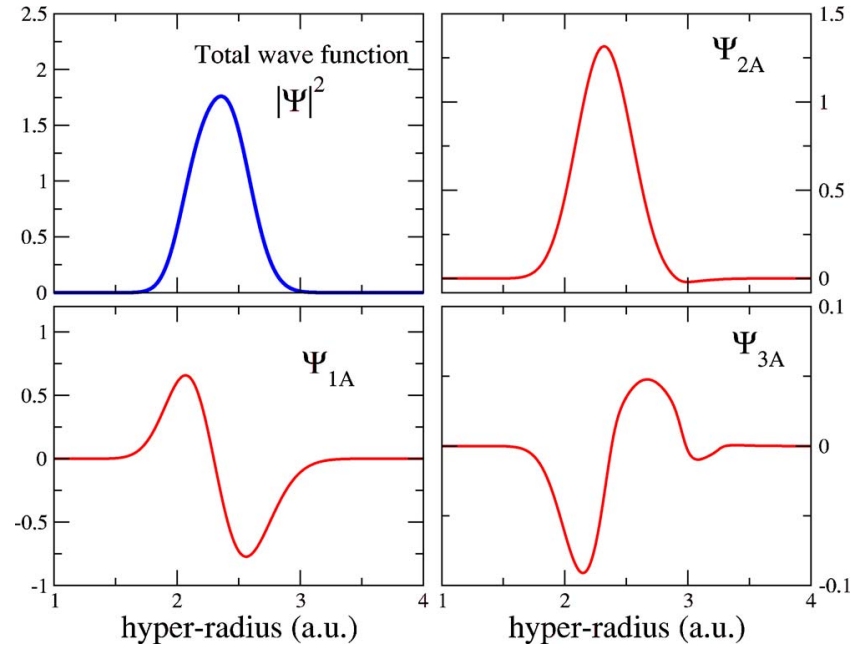

FIG. 6. (Color online) The figure shows the hyper-radial dependence $\psi_{a}(\rho)$ of the first excited $A$ vibrational wave function of $\mathrm{H}_{2} \mathrm{D}^{+}$ calculated using the SVD method. In the SVD method, vibrational eigenfunctions are multichannel [see Eq. (18)]. The upper left panel shows the probability amplitude of the first excited $A$ eigenfunction summed over all channels; the three other panels show contributions $\psi_{a}(\rho)$ of the first three adiabatic channels $(a=1,2,3)$ to the total eigenfunction.

of the nonadiabatic effects reduces the error from $20 \%$ to, at least, $1 \%$.

We have shown on the example of the isotopomers of $\mathrm{H}_{3}{ }^{+}$ that the hyperspherical coordinates allow us to treat properly all possible irreducible representations of the corresponding symmetry group. This can be useful in the problems where one has to select wave functions of a particular irreducible representation, not necessarily the ground state one, which is totally symmetric. An example is the three-body recombination processes in an ultracold gas of fermions: The ground rovibrational state of three identical fermions cannot be totally symmetric state. One has to consider states of other irreducible representations.

As discussed in the previous study by Fedorov et al. [66], the model three-boson system has a resonance. Currently, our present approach does not allow direct calculation of energies and widths of resonances. However, in the future we would like to adapt the approach for such calculations. A possible solution could be to use a complex absorbing poten-

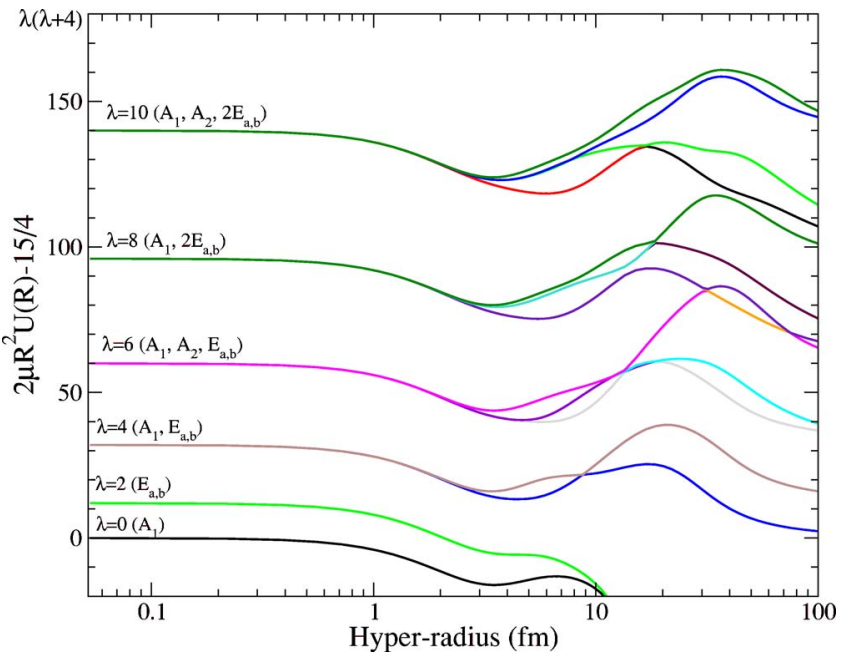

FIG. 7. (Color online) First several hyperspherical curves of the model three-boson system. The curves are scaled to give the eigenvalues of the grand angular momentum at small hyper-radii. The eigenvalues are $\lambda(\lambda+4)$ with $\lambda=0,2,4,6, \ldots$.

tial along $\rho$ or to use a complex scaling potential similar to the one employed in Ref. [66].

Future work should compare the accuracy of the present method with the diabatic-by-sector method developed by Launay and co-workers [23,34] for collision calculations and adapted by Willner [68] to loosely bound levels.

In conclusion, we have shown that the weakly bound states of different three-body systems can be effectively and uniformly treated using the hyperspherical coordinates and the mapping procedure employed along the hyper-radius. The advantage of the present method is that it can easily be implemented for calculation of vibrational wave functions, relevant to dynamics of cold molecules. The nonadiabatic hyperspherical effects seem to be always important and should be included into consideration.

\section{ACKNOWLEDGMENTS}

We acknowledge the Support of the Donors of the American Chemical Society Petroleum of Research Fund. This work has been partially supported by the National Science Foundation under Grant No. PHY-0427460, by an allocation of NCSA supercomputing resources (Project No. PHY040022).
[1] J. Doyle, B. Friedrich, R. Krems, and F. Masnou-Seeuws, Eur. Phys. J. D 31, 149 (2004).

[2] E. A. Donley, N. R. Claussen, S. T. Thompson, and C. E. Wieman, Nature (London) 417, 529 (2002).

[3] N. R. Claussen, E. A. Donley, S. T. Thompson, and C. E. Wieman, Phys. Rev. Lett. 89, 010401 (2002).

[4] J. Herbig, T. Kraemer, M. Mark, T. Weber, C. Chin, H.-C. Nägerl, and R. Grimm, Science 301, 1510 (2003).

[5] K. Xu, T. Mukaiyama, J. R. Abo-Shaeer, J. K. Chin, D. E. Miller, and W. Ketterle, Phys. Rev. Lett. 91, 210402 (2003).
[6] S. Dürr, T. Volz, A. Marte, and G. Rempe, Phys. Rev. Lett. 92, 020406 (2003).

[7] M. Greiner, C. A. Regal, and D. S. Jin, Nature (London) 426, 537 (2003).

[8] S. Jochim, M. Bartensein, A. Altmeyer, G. Hendl, C. Chin, S. Riedl, J. H. Denschlag, and R. Grimm, Science 302, 2101 (2003).

[9] M. W. Zwierlein, C. A. Stan, C. H. Schunck, S. M. F. Raupach, S. Gupta, Z. Hadzibabic, and W. Ketterle, Phys. Rev. Lett. 91, 250401 (2003). 
[10] K. E. Strecker, G. B. Partridge, and R. G. Hulet, Phys. Rev. Lett. 91, 080406 (2003).

[11] J. Cubizolles, T. Bourdel, S. J. J. M. F. Kokkelmans, G. V. Shlyapnikov, and C. Salomon, Phys. Rev. Lett. 91, 240401 (2003).

[12] S. Jochim, M. Bartenstein, A. Altmeyer, G. Hendl, C. Chin, J. H. Denschlag, and R. Grimm, Phys. Rev. Lett. 91, 240402 (2003).

[13] C. A. Regal, C. Ticknor, J. L. Bohn, and D. S. Jin, Nature (London) 424, 47 (2003).

[14] T. Bourdel, L. Khaykovich, J. Cubizolles, J. Zhang, F. Chevy, M. Teichmann, L. Tarruell, S. J. J. M. F. Kokkelmans, and C. Salomon, Phys. Rev. Lett. 93, 050401 (2004).

[15] A. J. Kerman, J. M. Sage, S. Sainis, T. Bergeman, and D. DeMille, Phys. Rev. Lett. 92, 033004 (2004).

[16] A. J. Kerman, J. M. Sage, S. Sainis, T. Bergeman, and D. DeMille, Phys. Rev. Lett. 92, 153001 (2004).

[17] D. Wang, J. Qi, M. F. Stone, O. Nikolayeva, B. Hattaway, S. D. Gensemer, H. Wang, W. T. Zemke, P. L. Gould, E. E. Eyler, and W. C. Stwalley, Eur. Phys. J. D 31, 165 (2004).

[18] D. Wang, J. Qi, M. F. Stone, O. Nikolayeva, H. Wang, B. Hattaway, S. D. Gensemer, P. L. Gould, E. E. Eyler, and W. C. Stwalley, Phys. Rev. Lett. 93, 243005 (2004).

[19] C. Chin, T. Kraemer, M. Mark, J. Herbig, P. Waldburger, H.-C. Nägerl, and R. Grimm, Phys. Rev. Lett. 94, 123201 (2005).

[20] N. Balakrishnan, R. C. Forrey, and A. Dalgarno, Phys. Rev. Lett. 80, 3224 (1998).

[21] N. Balakrishnan, A. Dalgarno, and R. C. Forrey, J. Chem. Phys. 113, 621 (2000).

[22] R. C. Forrey, N. Balakrishnan, A. Dalgarno, M. R. Haggerty, and E. J. Heller, Phys. Rev. Lett. 82, 2657 (1999).

[23] P. Soldán, M. T. Cvitas, J. M. Hutson, P. Honvault, and J.-M. Launay. Phys. Rev. Lett. 89, 153201 (2002).

[24] E. Bodo, F. A. Gianturco, and A. Dalgarno. Chem. Phys. Lett. 353, 127 (2002).

[25] M. T. Cvitas, P. Soldan, J. M. Hutson, P. Honvault, and J.-M. Launay, Phys. Rev. Lett. 94, 033201 (2005).

[26] M. T. Cvitas, P. Soldan, J. M. Hutson, P. Honvault, and J.-M. Launay, Phys. Rev. Lett. 94, 200402 (2005).

[27] E. Fattal, R. Baer, and R. Kosloff, Phys. Rev. E 53, 1217 (1996).

[28] E. Tiesinga, C. J. Williams, and P. S. Julienne, Phys. Rev. A 57, 4257 (1998).

[29] V. Kokoouline, O. Dulieu, R. Kosloff, and F. Masnou-Seeuws, J. Chem. Phys. 110, 9865 (1999).

[30] D. Lemoine, Chem. Phys. Lett. 210, 492 (2000).

[31] A. G. Borisov, J. Chem. Phys. 114, 7770 (2001).

[32] M. Nest and H.-D. Meyer, Chem. Phys. Lett. 352, 486 (2002).

[33] K. Willner, O. Dulieu, and F. Masnou-Seeuws, J. Chem. Phys. 120, 548 (2004).

[34] J.-M. Launay and M. Le Dourneuf, Chem. Phys. Lett. 163, 178 (1989).

[35] A. R. Cooper, S. Jain, and J. M. Hutson, J. Chem. Phys. 98, 2160 (1993).

[36] O. I. Tolstikhin, S. Watanabe, and M. Matsuzawa, J. Phys. B 29, L389 (1996).

[37] B. R. Johnson, J. Chem. Phys. 73, 5051 (1980).
[38] B. D. Esry, C. D. Lin, and C. H. Greene, Phys. Rev. A 54, 394 (1996).

[39] D. Blume and C. H. Greene, J. Chem. Phys. 113, 2145 (2000).

[40] V. Kokoouline and C. H. Greene, Phys. Rev. A 68, 012703 (2003).

[41] B. R. Johnson, J. Chem. Phys. 791916 (1983).

[42] R. Kosloff, in Dynamics of Molecules and Chemical Reactions, edited by R. H. Wyatt and J. Z. H. Zhang (Marcel Dekker, New York, 1996), p. 185.

[43] C. C. Marston and G. Balint-Kurti, J. Chem. Phys. 91, 3571 (1989).

[44] D. T. Colbert and W. H. Miller, J. Chem. Phys. 96, 1982 (1992).

[45] M. Monnerville and J. M. Robbe, J. Chem. Phys. 101-112, 7580 (1994).

[46] T. Cornelius and W. Glöckle, J. Chem. Phys. 85, 3906 (1986).

[47] V. Kokoouline, O. Dulieu, R. Kosloff, and F. Masnou-Seeuws, Phys. Rev. A 62, 032716 (2000).

[48] V. Kokoouline, O. Dulieu, and F. Masnou-Seeuws, Phys. Rev. A 62, 022504 (2000).

[49] B. Poirier and J. C. Light, J. Chem. Phys. 114, 6562 (2001).

[50] G. Katz, K. Yamashita, Y. Zeiri, and R. Kosloff, J. Chem. Phys. 116, 4403 (2002).

[51] V. Kokoouline, C. H. Greene, and B. D. Esry, Nature (London) 412, 891 (2001).

[52] V. Kokoouline and C. H. Greene, Phys. Rev. Lett. 90, 133201 (2003).

[53] I. Tuvi and Y. B. Band, J. Chem. Phys. 107, 9079 (1997).

[54] R. A. Aziz, V. P. S. Nain, J. S. Carley, W. L. Taylor, and G. T. McConville, J. Chem. Phys. 70, 4330 (1979).

[55] R. A. Aziz and M. J. Slaman, J. Chem. Phys. 94, 8047 (1991).

[56] E. Nielsen, D. V. Fedorov, and A. S. Jensen, J. Phys. B 31, 4085 (1998).

[57] B. J. McCall and T. Oka, J. Chem. Phys. 113, 3104 (2000).

[58] B. J. McCall, Philos. Trans. R. Soc. London, Ser. A 358, 2385 (2000).

[59] C. M. Lindsay and B. J. McCall, J. Mol. Spectrosc. 210, 60 (2001).

[60] O. L. Polyansky and J. Tennyson, J. Chem. Phys. 110, 5056 (1999).

[61] E. Cuervo-Reyes, J. Rubayo-Soneira, A. Aguado, M. Paniagua, C. Tablero, C. Sanz, and O. Roncero, Phys. Chem. Chem. Phys. 4, 6012 (2002).

[62] R. Jaquet, Spectrochim. Acta, Part A 58, 691 (2002).

[63] P. Schiffels, A. Alijah, and J. Hinze, Mol. Phys. 101, 175 (2003).

[64] W. Cencek, J. Rychlewski, R. Jaquet, and W. Kutzelnigg, J. Chem. Phys. 108, 2831 (1998).

[65] R. Jaquet, W. Cencek, W. Kutzelnigg, and J. Rychlewski, J. Chem. Phys. 108, 2837 (1998).

[66] D. V. Fedorov, E. Garrido, and A. S. Jensen, Few-Body Syst. 33, 153 (2003).

[67] J. Avery, Hyperspherical Harmonics: Applications in Quantum Theory (Kluwer Academic, Dordrecht, 1989).

[68] K. Willner, Ph.D. thesis, Université Paris XI, 2005 (unpublished). 